Portland State University

PDXScholar

Electrical and Computer Engineering Faculty

Publications and Presentations

$1-1-2005$

\title{
Effects of Focusing-Beam Field Variations on the Stability of Laser Oscillators
}

Pitak Chenkosol

Lee W. Casperson

Portland State University

Follow this and additional works at: https://pdxscholar.library.pdx.edu/ece_fac

Part of the Electrical and Computer Engineering Commons

Let us know how access to this document benefits you.

\section{Citation Details}

Pitak Chenkosol and Lee W. Casperson, "Effects of focusing-beam field variations on the stability of laser oscillators," J. Opt. Soc. Am. B 22, 1300-1308 (2005).

This Article is brought to you for free and open access. It has been accepted for inclusion in Electrical and Computer Engineering Faculty Publications and Presentations by an authorized administrator of PDXScholar. Please contact us if we can make this document more accessible: pdxscholar@pdx.edu. 


\title{
Effects of focusing-beam field variations on the stability of laser oscillators
}

\author{
Pitak Chenkosol* and Lee W. Casperson ${ }^{\dagger}$ \\ Department of Electrical and Computer Engineering and Department of Physics, Portland State University, \\ P.O. Box 751, Portland, Oregon 97207
}

Received April 30, 2004; revised manuscript received December 6, 2004; accepted January 4, 2005

\begin{abstract}
Semiclassical models for the dynamical behavior of single-longitudinal-mode, homogeneously broadened, unidirectional ring-laser oscillators have long been available. However, in most of these models the beam's cross section is assumed to be independent of longitudinal position in the laser, while in practical laser systems it is common for the diameter to vary with position. A model for lasers with focusing-beam field variations is developed here. Linear stability analysis shows that higher values of excitation are required to reach the type 1 instability threshold for lasers with longitudinal variations of the beam diameter than for the uniform planewave laser model. (C) 2005 Optical Society of America
\end{abstract}

OCIS codes: 270.3430, 270.3100, 020.1670, 140.3460.

\section{INTRODUCTION}

The spontaneous pulsation behavior of laser oscillators has been the subject of many studies. As a result of the nonlinear interactions of the cavity electric field with the laser medium, laser outputs may be stable, or they may exhibit undamped periodic or nonperiodic pulsations. The detailed form of the output depends on the values of the parameters that describe the operating conditions of the lasers. Most dynamical studies of lasers assume that the electric field in the cavity is in the form of a timedependent plane wave with infinite transverse extent. In real lasers, however, the electric field in the cavity always has either fixed or time-varying spatial variations. This is due to the nonuniform cavity losses and the finite dimensions of the resonator's mirrors or other components. In addition, the nonlinear interaction of the electric field with the laser medium can sometimes result in complex and possibly time-dependent transverse field patterns. More accurate laser models require the inclusion of an electric field with spatial variations.

There have been previous attempts at incorporating basic transverse field distributions into models for laser dynamics. However, most early studies were based on rateequation models, ${ }^{1,2}$ which are often insufficient for the study of spontaneous pulsation behavior. Of particular interest here, Globus et al. showed that the damping rate of relaxation oscillations in a rate-equation laser model is increased for a focusing-beam laser model in which the beam in the laser cavity is a function of longitudinal position. ${ }^{1}$ More general semiclassical models have revealed many other interesting results. In the case of laser oscillators, studies have also focused on the effect of the Gaussian ( $\mathrm{TEM}_{00}$ ) field on the dynamical behaviors of the laser systems. ${ }^{2-7}$ The effect of a saturable absorber on the linear stability of a laser with a Gaussian transverse profile has been studied by Horowicz et al. ${ }^{6}$ The instability of optically pumped lasers with Gaussian-shaped, radially varying gain profiles has been investigated by Smith and
Dykstra. ${ }^{8}$ They showed that the minimum value of instability threshold can be reached when the diameter of the pump beam is comparable to the diameter of the beam waist of the Gaussian cavity mode.

The early rate-equation results of Globus et al. ${ }^{1}$ and of Polloni and Svelto ${ }^{2}$ show that spatial variations of the electric field tend to stabilize the laser output. In a semiclassical study, Lugiato and Milani ${ }^{4}$ have further shown that the inclusion of only the fundamental-mode Gaussian $\left(\mathrm{TEM}_{00}\right)$ with constant spotsize in the laser model can eliminate the instability threshold for singlelongitudinal-mode, homogeneously broadened ring-laser oscillators. ${ }^{4}$ It has also been shown that the longitudinal field variations associated with a standing-wave model result in an increased threshold for laser instability. ${ }^{9}$ For the present stability criteria studies, we have developed a semiclassical model for a single-longitudinal-mode, homogeneously broadened, unidirectional ring-laser oscillator with a focusing-beam laser field. In this model, as in many practical laser oscillators, the beam diameter is a function of longitudinal position between the laser mirrors.

As noted in former stability studies ${ }^{9,10}$ two types of stability criteria may be distinguished, viz., type 1 and type 2 . In the present study, we focus only on the type 1 stability criterion, which corresponds to the minimum value of the excitation for which infinitesimal perturbations from the steady-state solution grow into oscillatory solutions. The type 2 stability criterion, which corresponds to the minimum value of the excitation for which large amplitude oscillatory solutions do not decay to the steady-state solutions, is not considered here. We perform linear stability analysis on the laser model we derive and then numerically calculate values of the real and imaginary parts of the complex rate constant for different values of the laser parameters. The sign of the real part of this complex rate constant determines whether the laser under study is stable. If the real part of the rate constant is negative, 
the infinitesimal perturbations from the steady state decay with time and the laser is stable. However, if the real part of the rate constant is positive the perturbations grow with time and the laser is unstable. The magnitude of this real part of the complex rate constant yields the decay rate (for a stable laser) or the growth rate (for an unstable laser) of the small perturbations. The imaginary part of the complex rate constant, on the other hand, gives an estimate of either the perturbation pulsation frequency for an unstable laser or the relaxation oscillation frequency of damped solutions for a stable laser.

The values of the rate constant derived here are graphically compared for the uniform and focusing-beam laser models. The comparisons show that the inclusion of wave focusing tends to raise the type 1 instability threshold compared to its value for the laser model with a uniform plane-wave field. Section 2 contains the general derivations of the focusing-beam model, and simplified versions are presented in Section 3. Linear stability analysis and numerical calculations of the values of the complex rate constant are presented in Section 4 for various values of the laser parameters. Graphical representations are given for the values of the real and imaginary parts of the complex rate constant as functions of realistic laser parameters. These rate constants in turn determine the type 1 instability threshold. The most familiar studies of spontaneous pulsations in homogeneously broadened laser oscillators all relate to gas lasers, including especially the $81.5-\mu \mathrm{m}$ ammonia far-infrared laser. ${ }^{11}$ To be specific the numerical calculations here are based on numbers appropriate for such an ammonia laser system.

\section{GENERAL MODEL}

The starting point for this analysis is a set of MaxwellSchrödinger equations that is similar to that used in a previous study of the spontaneous pulsations in ring-laser oscillators. ${ }^{12}$ The equations for the time dependence of the elements of the density matrix for single-longitudinalmode, homogeneously broadened ring-laser oscillators are

$$
\begin{aligned}
\frac{\partial \rho_{a b}(r, z, t)}{\partial t}= & -\left(i \omega_{0}+\gamma\right) \rho_{a b}(r, z, t)-i \frac{\mu}{\hbar} E(r, z, t) \\
& \times\left[\rho_{a a}(r, z, t)-\rho_{b b}(r, z, t)\right], \\
\frac{\partial \rho_{a a}(r, z, t)}{\partial t}= & \lambda_{a}(r, z, t)-\lambda_{a} \rho_{a a}(r, z, t)+\left[i \frac{\mu}{\hbar} E(r, z, t) \rho_{b a}(r, z, t)\right. \\
& + \text { c.c. }],
\end{aligned}
$$

$$
\begin{aligned}
\frac{\partial \rho_{b b}(r, z, t)}{\partial t}= & \lambda_{b}(r, z, t)-\gamma_{b} \rho_{b b}(r, z, t)+\gamma_{a b} \rho_{a a}(r, z, t) \\
& -\left[i \frac{\mu}{\hbar} E(r, z, t) \rho_{b a}(r, z, t)+\text { c.c. }\right]
\end{aligned}
$$

$$
\rho_{b a}(r, z, t)=\rho_{a b}^{*}(r, z, t),
$$

where the subscripts $a$ and $b$ denote the upper and lower laser levels, respectively, $\gamma_{a}$ and $\gamma_{b}$ are the total decay rates for these levels, $\gamma_{a b}$ is the rate of direct decays from level $a$ to level $b, \gamma=\left(\gamma_{a}+\gamma_{b}\right) / 2+\gamma_{\mathrm{ph}}$ is the decay rate for the off-diagonal elements with $\gamma_{\mathrm{ph}}$ the rate of phaseinterrupting collisions, $\lambda_{a}$ and $\lambda_{b}$ are the pumping rates, $\omega_{0}$ is the center frequency of the homogeneously broadened laser transition, and c.c. represents the complex conjugate of the preceding terms. The elements of the density matrix are assumed here to have a radial dependence, where $r$ is the radial coordinate.

To complete the semiclassical model, the Schrödingerequation-based density-matrix equations above must be joined by a Maxwell-equation-based equation for the electric field in the cavity. The wave equation for the electric field of a cylindrically symmetric, linearly polarized wave in a laser medium can be written in cylindrical coordinates as

$$
\begin{aligned}
& \nabla_{t}^{2} E(r, z, t)+\frac{\partial^{2} E(r, z, t)}{\partial z^{2}}-\mu_{1} \epsilon_{1} \frac{\partial^{2} E(r, z, t)}{\partial t^{2}}-\mu_{1} \sigma \frac{\partial E(r, z, t)}{\partial t} \\
& \quad=\mu_{1} \frac{\partial^{2} P(r, z, t)}{\partial t^{2}}
\end{aligned}
$$

where

$$
\nabla_{t}^{2}-\frac{\partial^{2}}{\partial r^{2}}+\frac{1}{r} \frac{\partial}{\partial r}
$$

is the transverse Laplacian operator. The permeability $\mu_{1}$ and permittivity $\epsilon_{1}$ are understood to include all of the magnetic and dielectric properties of the laser medium except for the polarization $P$, which is due to the lasing atoms or molecules. The finite value of the conductivity $\sigma$ accounts for losses in the cavity, which include absorption and scattering in the laser medium. In addition if the transmission of the output mirror is small, the coupling loss can also be accounted for by the same conductivity term. In this case all the losses in the resonator can be combined and then uniformly distributed along the resonator length. If this were not possible, one would have to consider separately the losses in the laser medium and at the output mirror. The macroscopic polarization $P(r, z, t)$ driving the electric field can be related to the off-diagonal density-matrix elements by

$P(r, z, t)=\frac{1}{2} P^{\prime}(r, z, t) \exp [i(k z-\omega t)]+$ c.c. $=\mu \rho_{a b}(r, z, t)+$ c.c.,

and thus

$$
\rho_{a b}(r, z, t)=\frac{P^{\prime}(r, z, t)}{2 \mu} \exp [i(k z-\omega t)] .
$$

Equations (1)-(5) form a complete set from which the time and space dependences of the electric field can be determined, subject to the boundary conditions at the resonator mirrors. The laser configuration for this study is shown in Fig. 1. The lengths of the laser medium and the 


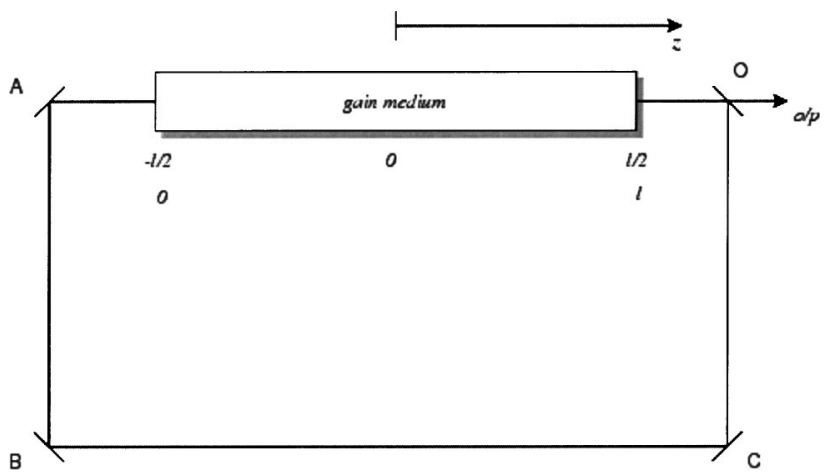

Fig. 1. Unidirectional ring cavity. The lengths of the laser medium and the cavity are $l$ and $L$, respectively. Mirrors A, B, and C have $100 \%$ reflectivity, whereas the output mirror $\mathrm{O}$ has reflectivity $R$.

resonator are $l$ and $L$, respectively. The reflection coefficient of the output mirror $O$ is $R$, where the other mirrors are assumed to have reflection coefficients of unity. The point $z=0$ is at the center of the gain medium as shown. The simplest solutions of Eqs. (1)-(5) are those that apply to unidirectional ring lasers in which both the electric field and the polarization are traveling waves.

Considering an empty cavity, i.e., $P(r, z, t)=0$, a solution of Eq. (5) can be written as

$$
E(r, z, t)=\frac{1}{2} E_{0} A(r, z) \exp \left[i\left(k_{0} z-\omega t\right)\right]+\text { c.c. },
$$

where $E_{0}$ is a constant and $A(r, z)$ is the transverse-fielddistribution function. With Eq. (9) substituted into Eq. (5), one can obtain [with $P(r, z, t)=0$ and $\sigma=0$ ]

$$
\nabla_{t}^{2} A(r, z)+i\left(2 k_{0}\right) \frac{\partial A(r, z)}{\partial z}=0 .
$$

A solution of Eq. (5) can be written in the general form

$$
\begin{aligned}
E(r, z, t) & =\frac{1}{2} E^{\prime}(r, z, t) \exp [i(k z-\omega t)]+\text { c.c. } \\
& =\frac{1}{2} E^{\prime}(z, t) A(r, z) \exp [i(k z-\omega t)]+\text { c.c. }
\end{aligned}
$$

This form of the electric field inside the cavity can represent a focusing beam. With the electric field as in Eq. (11), the macroscopic polarization $P(r, z, t)$ still has the same form as in Eq. (7). With Eqs. (7) and (11) substituted into Eq. (5), and use of the usual slowly varying amplitude approximations and the fact that $\mu_{1} \sigma \ll 2 \mu_{1} \epsilon_{1} \omega$, one obtains

$$
\begin{aligned}
\exp [ & i(k z-\omega t)]\left\{E^{\prime}(z, t)\left[\nabla_{t}^{2} A(r, z)+i(2 k) \frac{\partial A(r, z)}{\partial z}\right]\right. \\
& +E^{\prime}(z, t) A(r, z)\left(\mu_{1} \epsilon_{1} \omega^{2}-k^{2}+i \mu_{1} \sigma \omega\right) \\
& \left.+i 2 A(r, z)\left[k \frac{\partial E^{\prime}(z, t)}{\partial z}+\mu_{1} \epsilon_{1} \omega \frac{\partial E^{\prime}(z, t)}{\partial t}\right]\right\}+ \text { c.c. } \\
& =\exp [i(k z-\omega t)]\left[-\mu_{1} \omega^{2} P^{\prime}(r, z, t)\right]+\text { c.c. }
\end{aligned}
$$

At optical frequencies $k$ has a much smaller imaginary part than real part, and the first two terms on the left- hand side of Eq. (12) approximately cancel [see Eq. (10) with $k \approx k_{0}$ as in gas lasers], so one can rewrite it as

$$
\begin{gathered}
\exp [i(k z-\omega t)]\left\{E^{\prime}(z, t) A(r, z)\left(\mu_{1} \epsilon_{1} \omega^{2}-k^{2}+i \mu_{1} \sigma \omega\right)\right. \\
\left.+i 2 A(r, z)\left[k \frac{\partial E^{\prime}(z, t)}{\partial z}+\mu_{1} \epsilon_{1} \omega \frac{\partial E^{\prime}(z, t)}{\partial t}\right]\right\}+ \text { c.c. } \\
=\exp [i(k z-\omega t)]\left[-\mu_{1} \omega^{2} P^{\prime}(r, z, t)\right]+\text { c.c. }
\end{gathered}
$$

Multiplying Eq. (13) by $\exp [-i(k z-\omega t)]$ and then integrating the result over the cavity length $L$, we obtain

$$
\begin{aligned}
& \int_{-L / 2}^{L / 2}\left\{E^{\prime}(z, t) A(r, z)\left(\mu_{1} \epsilon_{1} \omega^{2}-k^{2}+i \mu_{1} \sigma \omega\right)\right. \\
& \left.\quad+i 2 A(r, z)\left[k \frac{\partial E^{\prime}(z, t)}{\partial z}+\mu_{1} \epsilon_{1} \omega \frac{\partial E^{\prime}(z, t)}{\partial t}\right]\right\} \mathrm{d} z \\
& \quad=\int_{-L / 2}^{L / 2}\left[-\mu_{1} \omega^{2} P^{\prime}(r, z, t)\right] \mathrm{d} z,
\end{aligned}
$$

where the terms that are multiplied by the factor $\exp [-i(k z-\omega t)] \exp \left[-i\left(k^{*} z-\omega t\right)\right]$ have been averaged out over a few optical cycles. The parameter $k^{*}$ is the complex conjugate of the propagation constant $k$.

As in Ref. 1 we now consider hypothetical lasers having uniformly distributed losses and focusing-beam fields. The dynamical equation for the complex amplitude of the electric field in this case can be obtained by assuming that the complex field amplitude $E^{\prime}$ is not a function of $z$ and the transverse-field-distribution function $A$ is not a function of the radial variable $r$. With these assumptions Eq. (14) reduces to

$$
\begin{aligned}
& E^{\prime}(t)\left(\mu_{1} \epsilon_{1} \omega^{2}-k^{2}+i \mu_{1} \sigma \omega\right) \int_{-L / 2}^{L / 2} A(z) \mathrm{d} z \\
& \quad+i 2 \mu_{1} \epsilon_{1} \omega \frac{\mathrm{d} E^{\prime}(t)}{\mathrm{d} t} \int_{-L / 2}^{L / 2} A(z) \mathrm{d} z \\
& \quad=-\mu_{1} \omega^{2} \int_{-L / 2}^{L / 2} P^{\prime}(z, t) \mathrm{d} z,
\end{aligned}
$$

where the real electric field and macroscopic polarization can be written, respectively, as

$$
\begin{aligned}
& E(z, t)=\frac{1}{2} E^{\prime}(t) A(z) \exp [i(k z-\omega t)]+\text { c.c. }, \\
& P(z, t)=\frac{1}{2} P^{\prime}(z, t) \exp [i(k z-\omega t)]+\text { c.c. }
\end{aligned}
$$

This reduced model allows us to address specifically the consequences of the focusing of the fields while setting aside other possible complications such as standing-wave fields, radial field variations, and high gain per pass, which have been considered in other studies.

The spatial field distribution $A(z)$ is assumed to have the form $A(z)=w_{0} / w(z)$ with $w(z)$ the $z$-dependent beam radius. In analogy with the focusing Gaussian beam we 
assume that in this simplified formalism, the beam radius is governed by the familiar relationship

$$
w(z)=w_{0}\left[1+\left(z / z_{0}\right)^{2}\right]^{1 / 2},
$$

where $w_{0}$ is the minimum beam radius and $z_{0}=\pi w_{0}^{2} / \lambda$ is the Rayleigh length. A plot of this focused-beam-envelope function $A(z)$ is shown in Fig. 2, together with other elements of the notation used here. The integral of $A(z)$ appearing in Eq. (15) and in subsequent equations can be performed analytically, but we have retained the integral form in the text for reasons of compactness.

Rearranging Eq. (15) one obtains

$$
\begin{gathered}
\frac{\mathrm{d} E^{\prime}(t)}{\mathrm{d} t}+\frac{\left(\mu_{1} \epsilon_{1} \omega^{2}-k^{2}+i \mu_{1} \sigma \omega\right)}{i 2 \mu_{1} \epsilon_{1} \omega} E^{\prime}(t)=i \frac{\omega}{2 \epsilon_{1}} \frac{\int_{-l / 2}^{l / 2} P^{\prime}(z, t) \mathrm{d} z}{\int_{-L / 2}^{L / 2} A(z) \mathrm{d} z}, \\
\frac{\mathrm{d} E^{\prime}(t)}{\mathrm{d} t}+\left(\frac{\sigma}{2 \epsilon_{1}}-i \frac{\omega^{2}-\Omega^{2}}{2 \omega}\right) E^{\prime}(t)=i \frac{\omega}{2 \epsilon_{1}} \frac{\int_{-l / 2}^{l / 2} P^{\prime}(z, t) \mathrm{d} z}{\int_{-L / 2}^{L / 2} A(z) \mathrm{d} z},
\end{gathered}
$$

where $\Omega=k /\left(\mu_{1} \epsilon_{1}\right)^{1 / 2}$ is the nondispersed cavity resonance frequency, i.e., the lasing frequency if the dispersion or real part of the polarization $P^{\prime}(z, t)$ were equal to zero. The $z$ integration on the polarization extends only over the medium length $l$. With the frequency simplifications $\omega \approx \Omega \approx \omega_{0}$, where $\omega_{0}$ is the center frequency of the laser transition, Eq. (20) can be rewritten as

$$
\frac{\mathrm{d} E^{\prime}(t)}{\mathrm{d} t}+\left[\frac{\sigma}{2 \epsilon_{1}}-i \gamma\left(y-y_{0}\right)\right] E^{\prime}(t)=i \frac{\omega_{0}}{2 \epsilon_{1}} \frac{\int_{-l / 2}^{l / 2} P^{\prime}(z, t) \mathrm{d} z}{\int_{-L / 2}^{L / 2} A(z) \mathrm{d} z}
$$

where the normalized frequencies $y$ and $y_{0}$ are defined as

$$
y=\left(\omega-\omega_{0}\right) / \gamma
$$

$$
y_{0}=\left(\Omega-\omega_{0}\right) / \gamma
$$

The macroscopic polarization in Eq. (17) can be related back to the off-diagonal element of the density matrix by

$$
P(z, t)=\frac{1}{2} P^{\prime}(z, t) \exp [i(k z-\omega t)]+\text { c.c. }=\mu \rho_{a b}(z, t)+\text { c.c. },
$$

and therefore

$$
\rho_{a b}(z, t)=\frac{P^{\prime}(z, t)}{2 \mu} \exp [i(k z-\omega t)] .
$$

If one substitutes $E(z, t)$ and $\rho_{a b}(z, t)$ from Eqs. (16) and (25), respectively, into Eqs. (1)-(4) one obtains the radially independent set

$$
\frac{\partial P^{\prime}(z, t)}{\partial t}=i\left(\omega-\omega_{0}\right) P^{\prime}(z, t)-\gamma P^{\prime}(z, t)-\frac{i \mu^{2}}{\hbar} E^{\prime}(t) A(z) D(z, t),
$$

$$
\begin{aligned}
\frac{\partial D(z, t)}{\partial t}= & \lambda_{d}(z, t)-\left(\frac{\gamma_{a}+\gamma_{a b}+\gamma_{b}}{2}\right) D(z, t) \\
& -\left(\frac{\gamma_{a}+\gamma_{a b}-\gamma_{b}}{2}\right) M(z, t)+\frac{i}{2 \hbar}\left[E^{\prime}(t) A(z) P^{\prime *}(z, t)\right. \\
& \left.-E^{\prime *}(t) A(z) P^{\prime}(z, t)\right]
\end{aligned}
$$

$$
\begin{aligned}
\frac{\partial M(z, t)}{\partial t}= & \lambda_{m}(z, t)-\left(\frac{\gamma_{a}-\gamma_{a b}-\gamma_{b}}{2}\right) D(z, t) \\
& -\left(\frac{\gamma_{a}-\gamma_{a b}+\gamma_{b}}{2}\right) M(z, t),
\end{aligned}
$$

where the population difference $D(z, t)=\rho_{a a}(z, t)-\rho_{b b}(z, t)$, the population sum $M(z, t)=\rho_{a a}(z, t)+\rho_{b b}(z, t)$, and the pump rates $\lambda_{d}(z, t)=\lambda_{a}(z, t)-\lambda_{b}(z, t)$ and $\lambda_{m}(z, t)=\lambda_{a}(z, t)$ $+\lambda_{b}(z, t)$ are introduced. Equations (21) and (26)-(28) can be rewritten in a normalized form as

$$
\frac{\partial P_{n}^{\prime}(z, t)}{\partial t}=-\gamma\left[(1-i y) P_{n}^{\prime}(z, t)+i E_{n}^{\prime}(t) A(z) D_{n}^{\prime}(z, t)\right],
$$

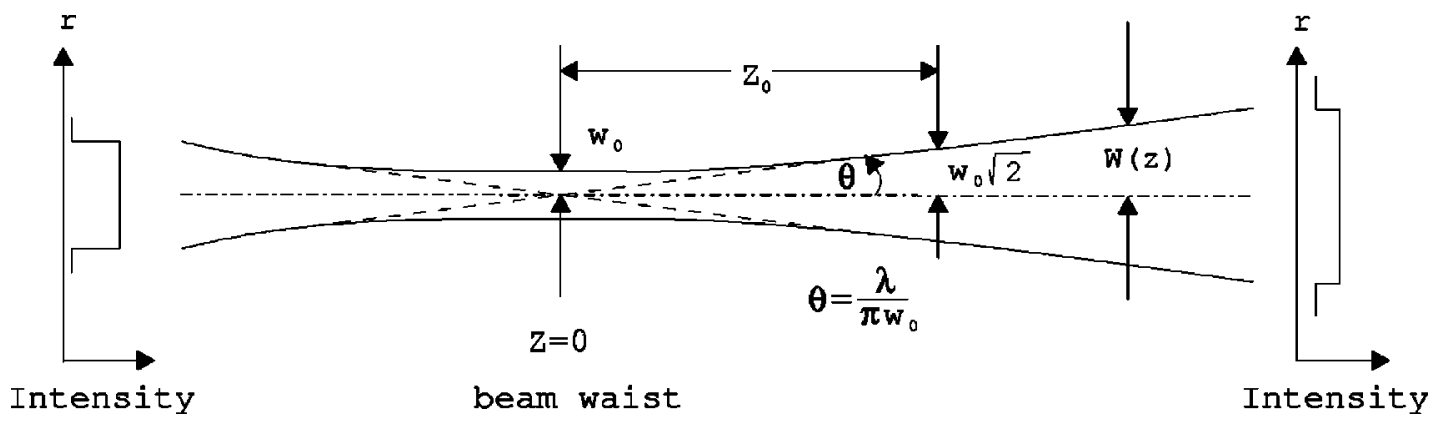

Fig. 2. Focused-beam function $A(z)$ and several notation definitions employed in the analysis. 


$$
\begin{aligned}
\frac{\partial D_{n}^{\prime}(z, t)}{\partial t}= & \lambda_{d n}^{\prime}(z, t)-\left(\frac{\gamma_{a}+\gamma_{a b}+\gamma_{b}}{2}\right) D_{n}^{\prime}(z, t) \\
& -\left(\frac{\gamma_{a}+\gamma_{a b}-\gamma_{b}}{2}\right) M_{n}^{\prime}(z, t)+i \gamma_{n} A(z) \\
& \times\left[E_{n}^{\prime}(t) P_{n}^{\prime *}(z, t)-E_{n}^{\prime *}(t) P_{n}^{\prime}(z, t)\right], \\
\frac{\partial M_{n}^{\prime}(z, t)}{\partial t}= & \lambda_{m n}^{\prime}(z, t)-\left(\frac{\gamma_{a}-\gamma_{a b}-\gamma_{b}}{2}\right) D_{n}^{\prime}(z, t) \\
& -\left(\frac{\gamma_{a}-\gamma_{a b}+\gamma_{b}}{2}\right) M_{n}^{\prime}(z, t),
\end{aligned}
$$$$
\frac{\mathrm{d} E_{n}^{\prime}(t)}{\mathrm{d} t}=-\gamma_{c}\left[1+i \delta\left(y-y_{0}\right)\right] E_{n}^{\prime}+i \gamma_{c} \frac{\int_{-l / 2}^{l / 2} P_{n}^{\prime}(z, t) \mathrm{d} z}{\int_{-L / 2}^{L / 2} A(z) \mathrm{d} z},
$$

where

$$
\begin{aligned}
E_{n}^{\prime}(t) & =\left(\frac{\gamma_{a}-\gamma_{a b}+\gamma_{b}}{2 \gamma \gamma_{a} \gamma_{b}}\right)^{1 / 2}\left(\frac{\mu}{\hbar}\right) E^{\prime}(t), \\
P_{n}^{\prime}(z, t) & =\left(\frac{\omega_{0}}{\sigma}\right)\left(\frac{\gamma_{a}-\gamma_{a b}+\gamma_{b}}{2 \gamma \gamma_{a} \gamma_{b}}\right)^{1 / 2}\left(\frac{\mu}{\hbar}\right) P^{\prime}(z, t), \\
D_{n}^{\prime}(z, t) & =\left(\frac{\omega_{0}}{\sigma}\right)\left(\frac{\mu^{2}}{\gamma \hbar}\right) D(z, t), \\
M_{n}^{\prime}(z, t) & =\left(\frac{\omega_{0}}{\sigma}\right)\left(\frac{\mu^{2}}{\gamma \hbar}\right) M(z, t), \\
\lambda_{d n}^{\prime}(z, t) & =\left(\frac{\omega_{0}}{\sigma}\right)\left(\frac{\mu^{2}}{\gamma \hbar}\right) \lambda_{d}(z, t), \\
\lambda_{m n}^{\prime}(z, t) & =\left(\frac{\omega_{0}}{\sigma}\right)\left(\frac{\mu^{2}}{\gamma \hbar}\right) \lambda_{m}(z, t),
\end{aligned}
$$

and $\gamma_{n}, \gamma_{c}$, and $\delta$ are defined as

$$
\begin{aligned}
& \gamma_{n}=\frac{\gamma_{a} \gamma_{b}}{\gamma_{a}-\gamma_{a b}+\gamma_{b}}, \\
& \gamma_{c}=\frac{1}{2 t_{c}}=\frac{\sigma}{2 \epsilon_{1}}, \\
& \delta=\frac{\gamma}{\gamma_{c}} .
\end{aligned}
$$

\section{SIMPLIFIED MODELS}

The laser Eqs. (29)-(32) form a general set that governs the behavior of homogeneously broadened ring-laser oscillators with focusing-beam field variations and uniformly distributed losses. However, these equations involve many variables and are not suitable for stability criteria study. Further simplifications can be introduced to reduce the number of equations and parameters without losing qualitative agreement between the theoretical study and experiments. First we will assume that the laser is operating exactly at cavity resonance at the center of the atomic gain profile $\omega=\Omega=\omega_{0}$. In normalized frequency units this is

$$
y=y_{0}=0 \text {. }
$$

Next we assume spatially and temporally independent pumping functions, i.e., $\lambda_{a}(z, t)=\lambda_{a}$ and $\lambda_{b}(z, t)=\lambda_{b}$. Another simplification involves a reduced model of the energy level diagram, viz. ${ }^{13}$

$$
\gamma_{b}=\gamma_{a}+\gamma_{a b}=\gamma_{d}
$$

With Eqs. (42) and (43) substituted into Eqs. (29)-(32), one obtains

$$
\begin{aligned}
\frac{\partial P_{n}^{\prime}(z, t)}{\partial t}= & -\gamma\left[P_{n}^{\prime}(z, t)+i E_{n}^{\prime}(t) A(z) D_{n}^{\prime}(z, t)\right], \\
\frac{\partial D_{n}^{\prime}(z, t)}{\partial t}= & -\gamma_{d}\left\{D_{n}^{\prime}(z, t)-\frac{\lambda_{d n}^{\prime}}{\gamma_{d}}-\frac{i}{2} A(z)\right. \\
& \left.\times\left[E_{n}^{\prime}(t) P_{n}^{\prime *}(z, t)-E_{n}^{\prime *}(t) P_{n}^{\prime}(z, t)\right]\right\}, \\
\frac{\mathrm{d} E_{n}^{\prime}(t)}{\mathrm{d} t}= & -\gamma_{c}\left[E_{n}^{\prime}(t)-i \frac{\int_{-l / 2}^{l / 2} P_{n}^{\prime}(z, t) \mathrm{d} z}{\int_{-L / 2}^{L / 2} A(z) \mathrm{d} z}\right] ;
\end{aligned}
$$

Eq. (31) becomes irrelevant under the above simplifications; Eqs. (44)-(46) can be rewritten in terms of new parameters $\widetilde{P}(z, t), \widetilde{D}(z, t), \widetilde{E}(t)$; and the threshold parameter $\mathfrak{R}$ is obtained in

$$
\begin{aligned}
\frac{\partial \widetilde{P}(z, t)}{\partial t}= & -\gamma[\widetilde{P}(z, t)+i \widetilde{E}(t) A(z) \widetilde{D}(z, t)], \\
\frac{\partial \widetilde{D}(z, t)}{\partial t}= & -\gamma_{d}\left\{\widetilde{D}(z, t)-\Re-\frac{i}{2} A(z)\left[\widetilde{E}(t) \widetilde{P}^{*}(z, t)\right.\right. \\
& \left.\left.-\widetilde{E}^{*}(t) \widetilde{P}(z, t)\right]\right\},
\end{aligned}
$$




$$
\frac{\mathrm{d} \widetilde{E}(t)}{\mathrm{d} t}=-\gamma_{c}\left[\widetilde{E}(t)-i \frac{\int_{-l / 2}^{l / 2} \widetilde{P}(z, t) \mathrm{d} z}{\int_{-l / 2}^{l / 2} A(z) \mathrm{d} z}\right],
$$

where

$$
\begin{gathered}
\widetilde{P}(z, t)=\frac{\int_{-l / 2}^{l / 2} A(z) \mathrm{d} z}{\int_{-L / 2}^{L / 2} A(z) \mathrm{d} z} P_{n}^{\prime}(z, t), \\
\widetilde{D}(z, t)=\frac{\int_{-l / 2}^{L / 2} A(z) \mathrm{d} z}{\int_{-L / 2}^{L / 2} A(z) \mathrm{d} z} D_{n}^{\prime}(z, t), \\
\widetilde{E}(t)=E_{n}^{\prime}(t), \\
\lambda_{d n}^{\prime}=\Re \lambda_{\text {th }}=\Re\left[\frac{\int_{-L / 2}^{L / 2} A(z) \mathrm{d} z}{\int_{-l / 2}^{l / 2} A(z) \mathrm{d} z}\right] \gamma_{d} .
\end{gathered}
$$

The parameter $\lambda_{\text {th }}$ is the threshold pump, which is the pumping rate required for the laser to start lasing. The threshold parameter $\mathfrak{R}$ is the ratio of the actual pump rate of the operating laser to the threshold pump rate. The polarization $\widetilde{P}(z, t)$ and the field amplitude $\widetilde{E}(t)$ in Eqs. (47)-(49) are complex quantities. They can be written in terms of their real and imaginary parts as $\widetilde{P}(z, t)$ $=\widetilde{P}_{r}(z, t)+i \widetilde{P}_{i}(z, t)$ and $\widetilde{E}(t)=\widetilde{E}_{r}(t)+i \widetilde{E}_{i}(t)$. With proper choices of the phases for $\widetilde{P}(z, t)$ and $\widetilde{E}(t)$ one can arbitrarily set $\widetilde{P}_{r}(z, t)=0, \widetilde{E}_{i}(t)=0$ and obtain from Eqs. (47)-(49) a real valued set:

$$
\begin{aligned}
& \frac{\partial \widetilde{P}_{i}(z, t)}{\partial t}=-\gamma\left[\widetilde{P}_{i}(z, t)+\widetilde{E}_{r}(t) A(z) \widetilde{D}(z, t)\right], \\
& \frac{\partial \widetilde{D}(z, t)}{d t}=-\gamma_{d}\left[\widetilde{D}(z, t)-\Re-\widetilde{E}_{r}(t) A(z) \widetilde{P}_{i}(z, t)\right], \\
& \frac{\mathrm{d} \widetilde{E}_{r}(t)}{\mathrm{d} t}=-\gamma_{c}\left[\widetilde{E}_{r}(t)+\frac{\int_{-l / 2}^{l / 2} \widetilde{P}_{i}(z, t) \mathrm{d} z}{\int_{-l / 2}^{l / 2} A(z) \mathrm{d} z}\right] .
\end{aligned}
$$

Considering Eqs. (54)-(56) at steady state, one can obtain

$$
\widetilde{P}_{i s}(z)=-\widetilde{E}_{r s} A(z) \widetilde{D}_{s}(z),
$$

$$
\widetilde{D}_{s}(z)=\Re+\widetilde{E}_{r s} A(z) \widetilde{P}_{i s}(z),
$$

$$
\widetilde{E}_{r s}=-\frac{\int_{-l / 2}^{l / 2} \widetilde{P}_{i s}(z) \mathrm{d} z}{\int_{-l / 2}^{l / 2} A(z) \mathrm{d} z} \gamma_{d},
$$

and steady-state solutions

$$
\begin{gathered}
\widetilde{D}_{s}(z)=\left[\frac{\Re}{1+\widetilde{E}_{r s}{ }^{2} A^{2}(z)}\right], \\
\widetilde{P}_{i s}(z)=-\left[\frac{\Re \widetilde{E}_{r s} A(z)}{1+\widetilde{E}_{r s}{ }^{2} A^{2}(z)}\right] .
\end{gathered}
$$

Next one can substitute $\widetilde{P}_{i s}(z)$ from Eq. (61) into Eq. (59) and obtain an equation for the steady-state electric field $\widetilde{E}_{r s}$ :

$$
\begin{aligned}
& \widetilde{E}_{r s}=-\frac{\int_{-l / 2}^{l / 2}\left[-\frac{\Re \widetilde{E}_{r s} A(z)}{1+\widetilde{E}_{r s}{ }^{2} A^{2}(z)}\right] \mathrm{d} z}{\int_{-l / 2}^{l / 2} A(z) \mathrm{d} z}, \\
& 1=\Re \frac{\int_{-l / 2}^{l / 2}\left[\frac{A(z)}{1+\widetilde{E}_{r s}{ }^{2} A^{2}(z)}\right] \mathrm{d} z}{\int_{-l / 2}^{l / 2} A(z) \mathrm{d} z}, \\
& 1=\Re \frac{\int_{0}^{l / 2}\left\{\frac{\left[1+\left(z / z_{0}\right)^{2}\right]^{1 / 2}}{\widetilde{E}_{r s}^{2}+1+\left(z / z_{0}\right)^{2}}\right\} \mathrm{d} z}{\int_{0}^{l / 2}\left[1+\left(z / z_{0}\right)^{2}\right]^{-1 / 2} \mathrm{~d} z},
\end{aligned}
$$

where again $A(z)=w_{0} / w(z)$ and Eq. (18) has been used for $w(z)$.

For a given set of $\mathfrak{R}$ and $w_{0}$ one can numerically solve Eq. (62) for the normalized steady-state electric field $\widetilde{E}_{r s}$. As above we introduce normalized decay rates $\delta=\gamma / \gamma_{c}, \rho$ $=\gamma_{d} / \gamma$ and a normalized time parameter $t^{\prime}=\gamma_{c} t$ and obtain from Eqs. (54)-(56) a new set:

$$
\begin{aligned}
& \frac{\partial \widetilde{P}_{i}\left(z, t^{\prime}\right)}{\partial t^{\prime}}=-\delta\left[\widetilde{P}_{i}\left(z, t^{\prime}\right)+\widetilde{E}_{r}\left(t^{\prime}\right) A(z) \widetilde{D}\left(z, t^{\prime}\right)\right], \\
& \frac{\partial \widetilde{D}\left(z, t^{\prime}\right)}{\partial t^{\prime}}=-\delta \rho\left[\widetilde{D}\left(z, t^{\prime}\right)-\Re-\widetilde{E}_{r}\left(t^{\prime}\right) A(z) \widetilde{P}_{i}\left(z, t^{\prime}\right)\right],
\end{aligned}
$$




$$
\frac{\mathrm{d} \widetilde{E}_{r}\left(t^{\prime}\right)}{\mathrm{d} t^{\prime}}=-\left[\widetilde{E}_{r}\left(t^{\prime}\right)+\frac{\int_{-l / 2}^{l / 2} \widetilde{P}_{i}\left(z, t^{\prime}\right) \mathrm{d} z}{\int_{-l / 2}^{l / 2} A(z) \mathrm{d} z}\right] .
$$

\section{LINEAR STABILITY ANALYSIS}

One of the main purposes of this study is to investigate the effects of nonuniform laser fields on the type 1 stability criteria of lasers with uniformly distributed losses. We first consider Eqs. (63)-(65) and linearize them by introducing the substitutions

$$
\begin{gathered}
\widetilde{P}_{i}\left(z, t^{\prime}\right)=\widetilde{P}_{i s}(z)+\widetilde{P}_{i}^{\prime}\left(z, t^{\prime}\right), \\
\widetilde{D}\left(z, t^{\prime}\right)=\widetilde{D}_{s}(z)+\widetilde{D}^{\prime}\left(z, t^{\prime}\right), \\
\widetilde{E}_{r}\left(t^{\prime}\right)=\widetilde{E}_{r s}+\widetilde{E}_{r}^{\prime}\left(t^{\prime}\right),
\end{gathered}
$$

where the primed quantities are assumed to be much smaller than the corresponding steady-state values. Substituting Eqs. (66)-(68) into Eqs. (63)-(65), respectively, we obtain

$$
\frac{\partial \widetilde{P}_{i}\left(z, t^{\prime}\right)}{\partial t^{\prime}}=-\delta\left\{\widetilde{P}_{i}^{\prime}\left(z, t^{\prime}\right)+A(z)\left[\widetilde{E}_{r s} \widetilde{D}^{\prime}\left(z, t^{\prime}\right)+\widetilde{D}_{s}(z) \widetilde{E}_{r}^{\prime}\left(t^{\prime}\right)\right]\right\},
$$

$$
\frac{\partial \widetilde{D}^{\prime}\left(z, t^{\prime}\right)}{\partial t^{\prime}}=-\delta \rho\left\{\widetilde{D}^{\prime}\left(z, t^{\prime}\right)-A(z)\left[\widetilde{E}_{r s} \widetilde{P}_{i}^{\prime}\left(z, t^{\prime}\right)+\widetilde{P}_{i s}(z) \widetilde{E}_{r}^{\prime}\left(t^{\prime}\right)\right]\right\}
$$

$$
\frac{\mathrm{d} \widetilde{E}_{r}\left(t^{\prime}\right)}{\mathrm{d} t^{\prime}}=-\left[\widetilde{E}_{r}\left(t^{\prime}\right)+\frac{\int_{-l / 2}^{l / 2} \widetilde{P}_{i}\left(z, t^{\prime}\right) \mathrm{d} z}{\int_{-l / 2}^{l / 2} A(z) \mathrm{d} z}\right],
$$

where we neglect the terms with products of primed quantities. Next we assume solutions of Eqs. (69)-(71) in the form

$$
\begin{aligned}
\widetilde{P}_{i}^{\prime}\left(z, t^{\prime}\right) & =\widetilde{P}_{i}^{\prime}(z) \exp \left(\lambda_{c} t^{\prime}\right), \\
\widetilde{D}^{\prime}\left(z, t^{\prime}\right) & =\widetilde{D}^{\prime}(z) \exp \left(\lambda_{c} t^{\prime}\right), \\
\widetilde{E}_{r}^{\prime}\left(t^{\prime}\right) & =\widetilde{E}_{r}^{\prime} \exp \left(\lambda_{c} t^{\prime}\right),
\end{aligned}
$$

where $\lambda_{c}$ is the complex rate constant. Substituting these forms into Eqs. (69)-(71), respectively, we get

$$
\begin{gathered}
\left(\lambda_{c}+\delta\right) \widetilde{P}_{i}^{\prime}(z)=-\delta A(z)\left[\widetilde{E}_{r s} \widetilde{D}^{\prime}(z)+\widetilde{D}_{s}(z) \widetilde{E}_{r}^{\prime}\right], \\
\left(\lambda_{c}+\delta \rho\right) \widetilde{D}^{\prime}(z)=\delta \rho A(z)\left[\widetilde{E}_{r s} \widetilde{P}_{i}^{\prime}(z)+\widetilde{P}_{i s}(z) \widetilde{E}_{r}^{\prime}\right],
\end{gathered}
$$

$$
\left(\lambda_{c}+1\right) \widetilde{E}_{r}^{\prime}=-\frac{\int_{-l / 2}^{l / 2} \widetilde{P}_{i}(z, t) \mathrm{d} z}{\int_{-l / 2}^{l / 2} A(z) \mathrm{d} z} .
$$

Combining Eqs. (75)-(77) with the steady-state solutions given in Eqs. (60) and (61) for $\widetilde{D}_{s}(z)$ and $\widetilde{P}_{i s}(z)$, respectively, one can write a relation for the complex rate constant $\lambda_{c}$ as

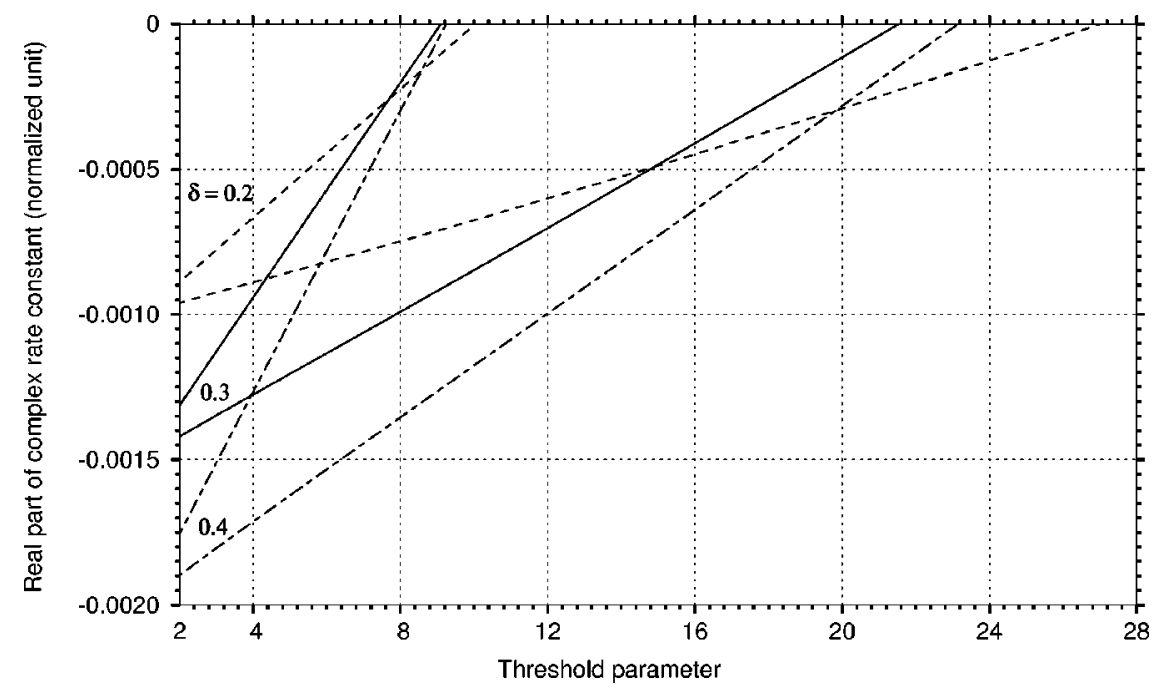

Fig. 3. Plots of the real part of the complex rate constant for lasers with focusing beam or uniform plane-wave fields as functions of the threshold parameter $\mathfrak{R}$ and decay ratio $\delta$. These are obtained by numerically solving Eq. (78), where the minimum spotsize of the field in the uniform plane-wave case is set to a very large number. The values of the other laser parameters are $w_{0}=0.004 \mathrm{~m}, l=1.5 \mathrm{~m}, \rho$ $=0.01$, and $\lambda=81.5 \mu \mathrm{m}$. The lower curve in each pair is for the focusing-beam field. 


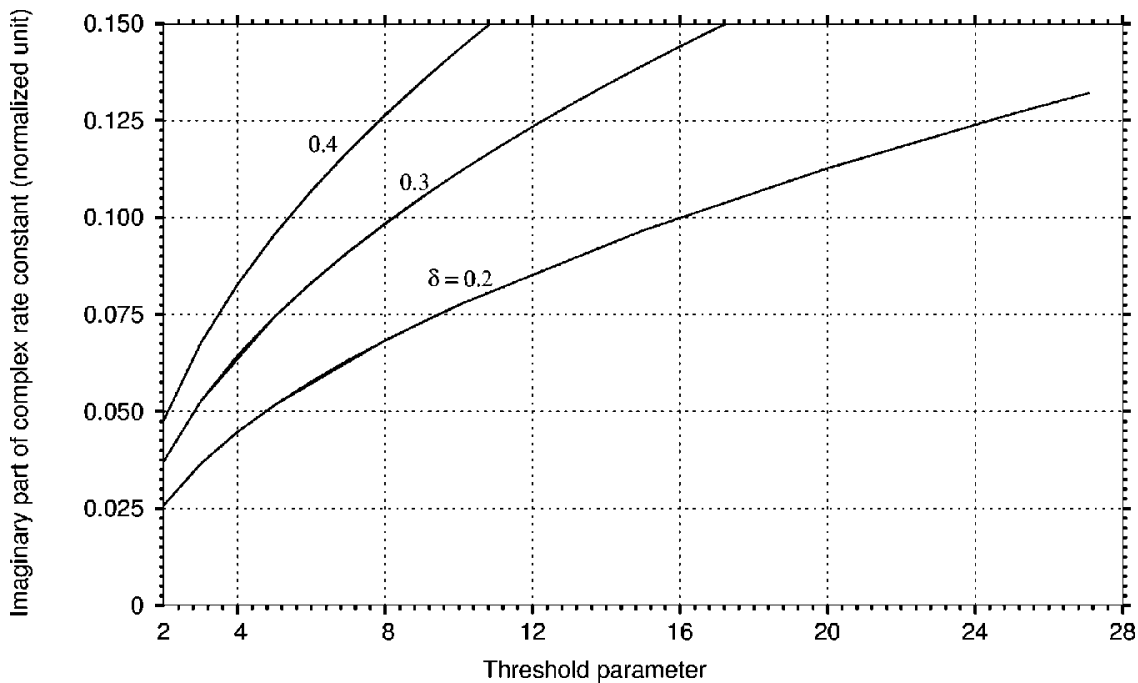

Fig. 4. Plots of the imaginary part of the complex rate constant for lasers with focusing-beam or uniform plane-wave fields as functions of the threshold parameter $\Re$ and decay ratio $\delta$. These are obtained by numerically solving Eq. (78), where the minimum spotsize of the field in the uniform plane-wave case is set to a very large number. The values of the other laser parameters are $w_{0}=0.004 \mathrm{~m}, l=1.5 \mathrm{~m}$, $\rho=0.01$, and $\lambda=81.5 \mu \mathrm{m}$

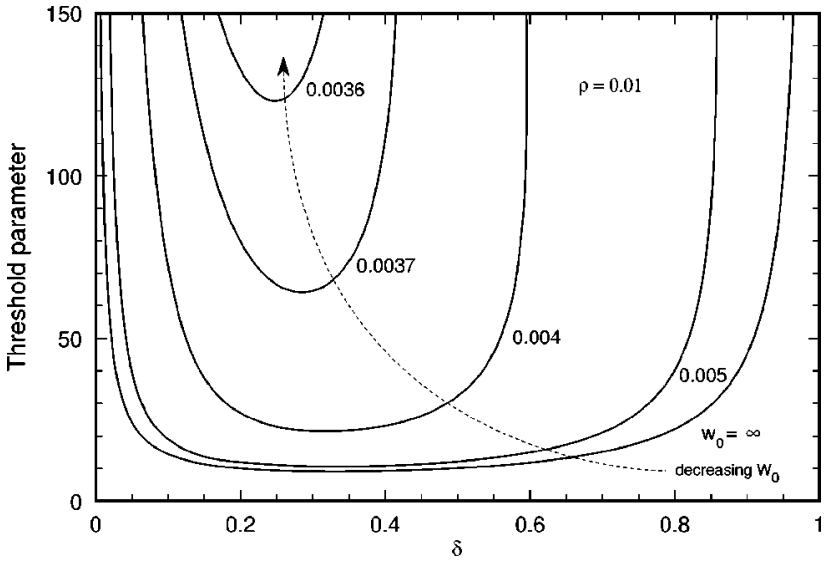

Fig. 5. Plots of the type 1 stability boundaries for lasers with focusing-beam and uniform plane-wave fields with $l=1.5 \mathrm{~m}, \rho$ $=0.01$, and $\lambda=81.5 \mu \mathrm{m}$, and values of the minimum spotsize $w_{0}$ as labeled. The lowest curve is the result from the uniform planewave model, i.e., $w_{0}$ is infinitely large. ${ }^{9}$

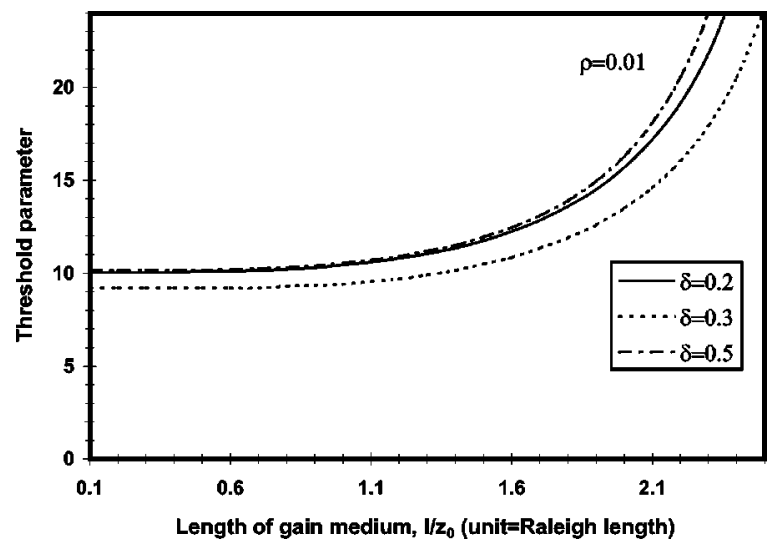

Fig. 6. Plots of the type 1 stability boundaries for lasers with focusing beams as functions of normalized length of the laser gain medium and the decay ratio $\delta$. The length of the gain medium is normalized to the Rayleigh length $z_{0}$. The values of the other laser parameters are $w_{0}=0.004 \mathrm{~m}, l=1.5 \mathrm{~m}, \rho=0.01$, and $\lambda=81.5 \mu \mathrm{m}$.

$$
\begin{aligned}
\lambda_{c}= & -1+\frac{\delta \Re}{\int_{0}^{l / 2} A(z) \mathrm{d} z} \\
& \times \int_{0}^{l / 2} \frac{A(z)\left\{\left(\lambda_{c}+\delta \rho\right)-\delta \rho\left[\widetilde{E}_{r s} A(z)\right]^{2}\right\} \mathrm{d} z}{\left\{1+\left[\widetilde{E}_{r s} A(z)\right]^{2}\right\}\left\{\left(\lambda_{c}+\delta \rho\right)\left(\lambda_{c}+\delta\right)+\delta^{2} \rho\left[\widetilde{E}_{r s} A(z)\right]^{2}\right\}},
\end{aligned}
$$

where again $A(z)=w_{0} / w(z)=\left[1+\left(z / z_{0}\right)^{2}\right]^{-1 / 2}$. Equation (78) has been numerically solved for the real and imaginary parts of $\lambda_{c}$ for different values of $z_{0}, \Re, \delta$, and $\rho$. The other laser parameters are specified as $w_{0}=0.004 \mathrm{~m}, l=1.5 \mathrm{~m}$, $\rho=0.01$, and $\lambda=81.5 \mu \mathrm{m}$; these values are not unreasonable for an ammonia laser. The results are shown in Figs. 3 and 4, where we also include the corresponding results from the familiar uniform plane-wave model, which can be obtained by solving Eq. (78) with an infinitely large minimum spotsize $w_{0}$.

A negative value of the real part of the complex rate constant, shown in Fig. 3, gives the damping rate of the relaxation oscillations, and a more negative value indicates more damping. The results show that, with the same set of parameters $z_{0}, \delta$, and $\rho$, larger values of the threshold parameter $\mathfrak{R}$ are required in the case of the focusing-beam, plane-wave field to reach the type 1 instability boundary compared with those values in the uniform plane-wave model. ${ }^{10}$ Near threshold, $\mathfrak{R}=1$, the small-perturbation damping rate for the focusing-beam and plane-wave models are approximately equal. The imaginary part of the complex rate constant, shown in Fig. 4, gives the relaxation oscillation frequency of a laser that is stable when subjected to a small perturbation. Each curve in Fig. 4 is actually two curves in close proximity.

A comparison of the type 1 stability boundaries for lasers with focusing-beam and uniform plane-wave fields is shown in Fig. 5. One can clearly observe that as the value 
of the minimum spotsize of a focusing beam is decreased, a higher value of threshold parameter is required to reach the type 1 instability. The lowest curve in the figure corresponds to the case of a laser with a uniform plane-wave field. This is related to the rate-equation result in Ref. 1 where it was shown that the damping of the relaxation oscillations in a laser with a focusing plane-wave field is stronger than that of a laser with a uniform plane-wave field. In addition, as shown in Fig. 5, the region where the laser is unstable is narrower when the value of the minimum spotsize of the focusing beam is smaller.

The stabilization effect of the focused-plane-wave field is further illustrated in Fig. 6. In this figure, we show three instability threshold curves as a function of the normalized length of the gain medium for three different values of the normalized decay rate $\delta$. The corresponding values of $\rho$ and minimum spotsize $w_{0}$ are 0.01 and $0.004 \mathrm{~m}$, respectively. The length of the gain medium shown in the horizontal axis is normalized to the Rayleigh length, i.e., normalized length $=1$ corresponds to the case when the physical length of the laser gain medium equals the Rayleigh length.

At any value of minimum spotsize, the region of the gain medium within the Rayleigh length will interact with a rather uniform-spotsize electric field where the beam focusing effect is minimal. This is comparable with the situation in plane-parallel resonator lasers. Beyond the Rayleigh length, however, the gain medium can interact more with the varying intensity region of the focused beam profile. The results emphasize that the focusing of the beam has a stabilizing effect by raising the selfpulsing instability threshold.

\section{CONCLUSION}

In this study, we have developed a semiclassical model for homogeneously broadened, unidirectional, ring-laser oscillators with focusing-beam distributions. This model can be used as a starting point for thorough dynamical studies of this type of laser. Linear stability analysis has also been performed on the simplified version of the model when the laser is assumed to be operated at exact cavity resonance and line-center tuning. Other parameter values have been chosen to correspond to the $81.5-\mu \mathrm{m}$ ammonia laser, which has been important in experimental studies of spontaneous pulsations in homogeneously broadened laser oscillators. The results show for the first time that a higher value of the threshold parameter is re- quired to reach the type 1 instability threshold in a focusing-beam laser oscillator than in a uniform planewave laser.

*Present address, Laser Technology Group, Maxim Integrated Products, 7250 Northwest Evergreen Parkway, Hillsboro, Oregon 97124.

${ }^{\dagger}$ Present address, Department of Electrical and Computer Engineering and the Center for Optoelectronics and Optical Communications of The University of North Carolina at Charlotte, 9201 University City Boulevard, Charlotte, North Carolina 28223.

\section{REFERENCES}

1. M. E. Globus, Yu. V. Naboikin, A. M. Ratner, I. A. RomKrichevskaya, and Yu. A. Tiunov, "Stationary generation in an optical resonator with lenses," Sov. Phys. JETP 25, 562-567 (1967).

2. R. Polloni and O. Svelto, "Static and dynamic behavior of a single-mode Nd-YAG laser," IEEE J. Quantum Electron. QE-4, 481-485 (1968).

3. L. A. Lugiato, R. J. Horowicz, G. Strini, and L. M. Narducci, "Instabilities in passive and active optical systems with a Gaussian transverse intensity profile," Phys. Rev. A 30, 1366-1376 (1984).

4. L. A. Lugiato and M. Milani, "Disappearance of laser instabilities in a Gaussian cavity mode," Opt. Commun. 46, 57-60 (1983).

5. L. A. Lugiato, F. Prati, L. M. Narducci, P. Ru, J. R. Tredicce, and D. K. Bandy, "Role of transverse effects in laser instabilities," Phys. Rev. A 37, 3847-3866 (1988).

6. R. J. Horowicz, L. A. Lugiato, and G. Strini, "Steady-state and stability analysis for a laser with a saturable absorber with a Gaussian transverse profile," Z. Phys. B: Condens. Matter 58, 71-78 (1984).

7. L. A. Lugiato, F. Prati, D. K. Bandy, L. M. Narducci, P. Ru, and J. R. Tredicce, "Low threshold instabilities in unidirectional ring lasers," Opt. Commun. 64, 167-171 (1987).

8. C. P. Smith and R. Dykstra, "Lorenz like chaos in a Gaussian mode laser with a radially dependent gain," Opt. Commun. 117, 107-110 (1995).

9. P. Chenkosol and L. W. Casperson, "Spontaneous coherent pulsations in standing-wave laser oscillators: stability criteria for homogeneous broadening," J. Opt. Soc. Am. B 10, 817-826 (1993).

10. L. W. Casperson, "Spontaneous coherent pulsations in ringlaser oscillators: stability criteria," J. Opt. Soc. Am. B 2, 993-997 (1985).

11. See, for example, C. O. Weiss, W. Klische, P. S. Ering, and M. Cooper, "Instabilities and chaos of a single-mode $\mathrm{NH}_{3}$ ring laser," Opt. Commun. 52, 405-408 (1985).

12. L. W. Casperson, "Spontaneous coherent pulsations in ringlaser oscillators," J. Opt. Soc. Am. B 2, 62-72 (1985).

13. L. W. Casperson, "Spontaneous coherent pulsations in ringlaser oscillators: simplified models," J. Opt. Soc. Am. B 2, 73-80 (1985). 